\title{
Human endometrial angiogenesis
}

\author{
Caroline E. Gargett and Peter A. W. Rogers \\ Monash University Department of Obstetrics and Gynecology, Monash Medical Centre, \\ 246 Clayton Road, Clayton, Victoria, 3168, Australia
}

\begin{abstract}
Angiogenesis is the development of new microvessels from existing vessels, a process that involves microvascular endothelial cells. Physiological angiogenesis rarely occurs in adults except in the ovary and endometrium during the reproductive life of females. Angiogenesis occurs by sprouting and non-sprouting mechanisms. Since endothelial sprouts are not observed in human endometrium, we hypothesized that non-sprouting mechanisms such as intussusception and elongation are involved in endometrial angiogenesis. The demand for angiogenesis differs spatially and temporally in the endometrium: angiogenesis occurs in the basalis layer during menstruation and in the functionalis and subepithelial capillary plexus during the proliferative and early secretory stages. Most studies have failed to demonstrate a link between expression of endometrial angiogenic factors and new vessel growth. However, we demonstrated recently a strong relationship between vascular endothelial growth factor (VEGF) immunolocalized in intravascular neutrophils and endothelial cell proliferation in each of the subepithelial capillary plexus, functionalis and basalis regions of the human endometrium. Our data also indicate that focal neutrophil VEGF has a role in the development of the subepithelial capillary plexus and functionalis microvessels during the proliferative phase of the menstrual cycle. We propose that neutrophils are an intravascular source of VEGF for vessels that undergo angiogenesis by intussusception and elongation.
\end{abstract}

Angiogenesis is the process by which new microvessels develop from existing blood vessels. Normal physiological angiogenesis occurs during fetal growth and development, but rarely occurs in adults except during episodes of wound healing and in the female reproductive tract. Throughout the reproductive life of females, angiogenesis occurs regularly in the corpus luteum and the endometrium as part of the rapid growth and regression that occurs in these tissues during the menstrual cycle (Torry and Torry, 1997; Smith, 1998; Rogers and Gargett, 1999). Pathological angiogenesis occurs in conditions such as malignancy, chronic inflammatory disorders (for example rheumatoid arthritis), endometriosis and diabetic retinopathy (Hanahan and Folkman, 1996).

This article briefly describes the mechanisms involved in angiogenesis and how the process is regulated, with particular reference to the human endometrium. Our hypotheses on the spatial and temporal relationships between angiogenesis and events associated with different stages of the menstrual cycle will be outlined. We will also describe some of our most recent observations and suggest a novel hypothesis on the regulation of angiogenesis in human endometrium during the proliferative phase of the menstrual cycle.

Email: caroline.gargett@med.monash.edu.au

\section{Mechanisms of angiogenesis}

The microvascular endothelial cells of the smallest vessels or capillaries are involved in angiogenesis. New vessel growth can occur by four different mechanisms in the adult: sprouting, intussusception, vessel elongation and incorporation of circulating endothelial progenitor cells into growing vessels (Fig. 1). Classical angiogenesis or sprouting involves a series of steps commencing with the activation of endothelial cells, which release proteases to break down the surrounding basement membrane. Endothelial cells then migrate towards the angiogenic stimulus, proliferate and coalesce to form patent tubules (Risau, 1997). A new basement membrane is deposited and the vessel is stabilized by the recruitment of pericytes and, in the larger vessels, smooth muscle cells. This process is mediated by angiopoietin-1 binding to endothelial Tie-2 receptors (Hanahan, 1997). Sprouting angiogenesis is important when neovascularization of an avascular tissue occurs, such as during the rapid growth of the corpus luteum after ovulation (Fig. 1a) and when tumours become angiogenic and recruit host vasculature. Intussusception is the process in which the lumen of a vessel is divided into two internally as proliferating endothelial cells migrate inwards, producing a network of interlocking vessels or an arcade of parallel vessels (Fig. 1c), as occurs in the developing lung (Risau, 1997). Elongation and vessel widening proba- 


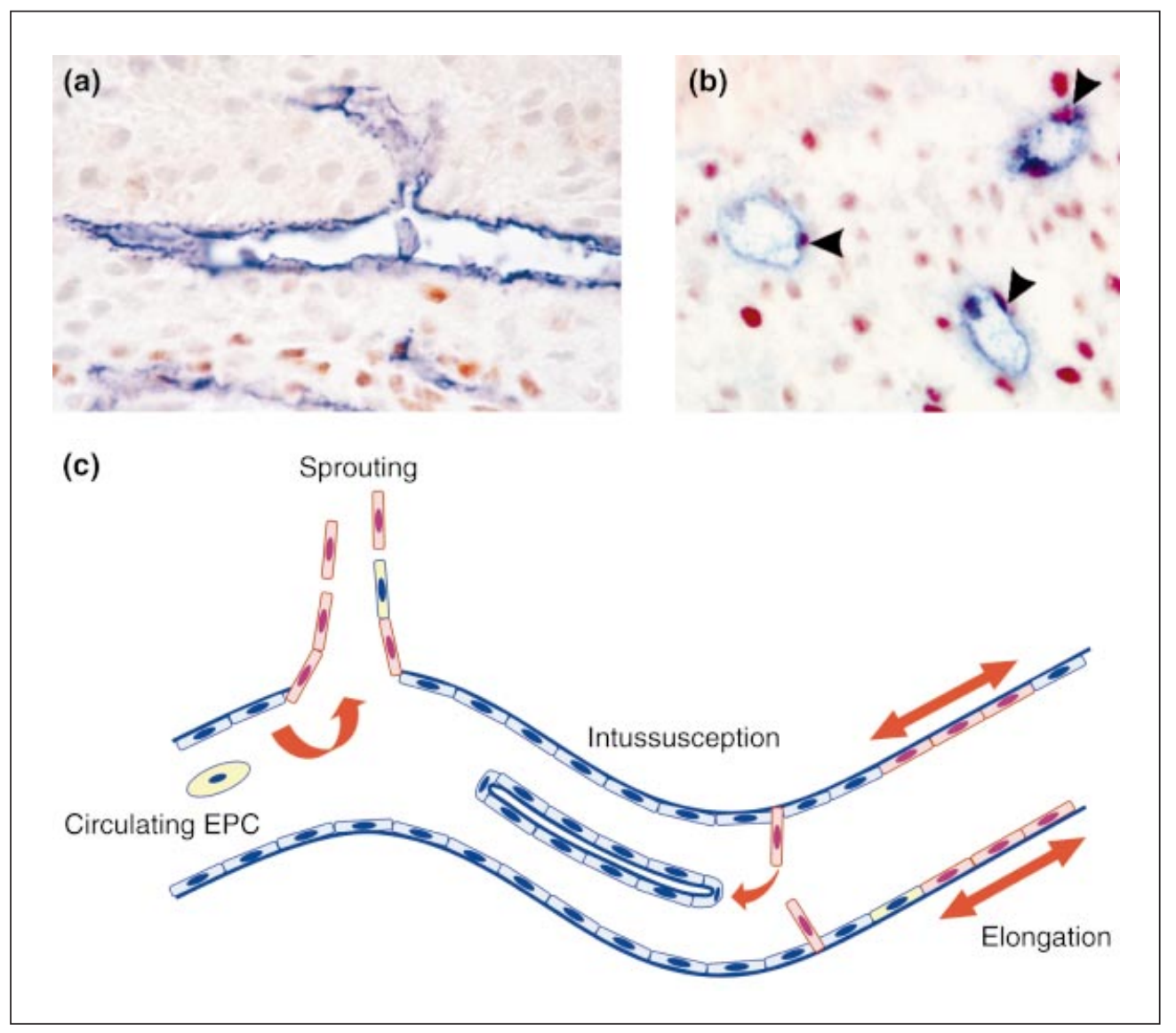

Fig. 1. Mechanisms of angiogenesis. Human ovary (a) and endometrium (b) double immunostained with CD34 (blue) to highlight endothelial cells and proliferating cell nuclear antigen (PCNA) (red) to highlight proliferating cells. Proliferating endothelial cells are shown by arrowheads. (a) Endothelial cells sprouting into the avascular granulosa layer of the ovary immediately after the LH surge and (b) endometrial vessel profiles containing proliferating endothelial cells. (c) Schematic representation of the four mechanisms of angiogenesis: sprouting, intussusception, elongation and incorporation of circulating endothelial progenitor cells (EPC). Quiescent endothelial cells are shown in blue, proliferating endothelial cells in red and endothelial progenitor cells in yellow.

bly occur in growing tissues as existing vessels constantly restructure in response to the metabolic demands of the surrounding cells, a process also known as remodelling or pruning (Fig. 1b,c) (Risau, 1997). Recently, a small proportion of circulating mononuclear cells were identified as endothelial cell progenitors, which have the capacity to incorporate into growing but not quiescent vessels (Fig. 1c) (Asahara et al., 1999).

\section{Regulation of angiogenesis}

Angiogenesis is an incompletely understood and complex process which is tightly regulated by numerous angiogenic promoters and inhibitors. In quiescent endothelium, these promoters and inhibitors are in balance. During an episode of vessel growth, the balance tips in favour of the promoters, whereas cessation of angiogenesis and vessel regression occur when the balance swings in favour of the inhibitors (Hanahan and Folkman, 1996). Vascular endothelial growth factor (VEGF) is one of the major regulators of angiogenesis, and its important role has been demonstrated in developmental, physiological and pathological angiogenesis (reviewed in Ferrara and Davis-Smyth, 1997). The angiogenic action of VEGF is mediated through binding to the tyrosine kinase receptor, VEGFR-2 (KDR), on endothelial cells. Although VEGF also binds to two other receptors, VEGFR-1 (flt-1) and neuropilin-1, the role of these receptors in angiogenesis appears to be minimal.

\section{Angiogenesis in the endometrium}

The demand for vessel growth and regression in human endometrium differs spatially and temporally during the sequence of events that occur during the menstrual cycle. There are three distinct stages when angiogenesis occurs: during menstruation for repair of the vascular bed, during 
the rapid endometrial growth of the proliferative phase and during the secretory stage when spiral arterioles show significant growth and coiling (Rogers and Gargett, 1999). A subepithelial capillary plexus also develops during the menstrual cycle. Blood flow through this plexus reaches a maximum during the early and mid-secretory phases in preparation for the implanting embryo (Gannon et al., 1997). Spatially, these angiogenic events occur in different regions or zones of the endometrium. Post-menstrual repair occurs in the superficial layer of the remaining basalis. Proliferative phase vascular growth occurs throughout the functionalis in which, under the influence of oestrogen, the endometrium approximately quadruples in thickness (Rogers and Gargett, 1999). The growth of spiral arterioles also occurs in the functionalis layer, whereas the capillary plexus which is supplied by the arterioles develops just below the luminal epithelial surface. Thus, unlike most vascular beds, which maintain a constant structure and function throughout life, the endometrial vasculature grows and regresses during the menstrual cycle and therefore provides a dynamic model for the study of physiological angiogenesis (Rogers and Gargett, 1999).

\section{Mechanisms involved in endometrial angiogenesis}

Several immunohistochemical studies on endothelial cell proliferation indicate that classical sprouting angiogenesis does not occur in human endometrium, since proliferating endothelial cells are always identified within vessel profiles and sprouts have not been observed (Fig. 1b) (reviewed in Rogers and Gargett, 1999). Furthermore, the immunohistochemical identification of $\alpha_{v} \beta_{3}$ integrin, a marker of sprouting endothelium, has been observed only within existing vessel profiles (Hii and Rogers, 1998). Therefore we hypothesized that vessel growth in human endometrium occurs by non-sprouting mechanisms. Specifically, we hypothesized that angiogenesis in functionalis endometrium during the proliferative phase occurs by elongation, whereas the complex, highly branched subepithelial capillary plexus develops by an intussusceptive mechanism during the proliferative and early secretory stages. The spiral arterioles grow by elongation throughout the menstrual cycle, but should be considered separately since arteriogenesis may be different from angiogenesis. Whether circulating endothelial progenitors have a role in human endometrial angiogenesis is yet to be determined, although there is evidence for such a role in a mouse model (Asahara et al., 1999).

\section{Regulation of endometrial angiogenesis}

Although the overall control of endometrial growth and regression is regulated primarily by circulating concentrations of oestrogen and progesterone, the role of sex steroids in endometrial angiogenesis is less clear. Most studies have failed to demonstrate oestrogen or progesterone receptors in human endometrial endothelium, with the exception of a recent study by Iruela-Arispe et al. (1999). Endothelial cell proliferation indices also vary widely throughout the menstrual cycle, showing no relationship to the stage of the cycle (Rogers and Gargett, 1999). It is likely that the numerous angiogenic promoters that have been identified in human endometrium regulate angiogenic events. Although, to date, little information has been published on the presence of angiogenesis inhibitors in human endometrium, many precursors of known angiogenesis inhibitors have been demonstrated in endometrium (reviewed in Smith, 1998; Rogers and Gargett, 1999).

Most work to date on endometrial angiogenic factors has focused on VEGF (Torry and Torry, 1997; Smith, 1998; Rogers and Gargett, 1999). Attempts have been made to relate VEGF mRNA and protein expression in human endometrium to stages of the menstrual cycle (Torry and Torry, 1997; Rogers and Gargett, 1999). These studies have produced conflicting results, although it is now clear that the bulk of endometrial VEGF is glandular in origin (Gargett et al., 1999). Most glandular VEGF is secreted apically (Hornung et al., 1998) and is unlikely to have an angiogenic role in human endometrium, since VEGF is also found in secretions from other non-angiogenic tissues (for example saliva, breast milk, semen). Few studies have examined VEGF expression in human endometrium in relation to known markers of angiogenesis, such as proliferating endothelial cells. In the first such study, we reported that neither total glandular nor total stromal VEGF production correlated with endometrial endothelial cell proliferation (Gargett et al., 1999). However, foci of VEGF immunostaining much more intensely than that of glands or stromal fibroblasts has been observed in the endometrial stromal compartment, particularly during the proliferative stage, and some of this VEGF was associated with blood vessels (Torry and Torry, 1997; Bausero et al., 1998; Gargett et al., 1999). In addition, during the proliferative phase, the proportion of capillaries expressing VEGFR-2 is much higher than in the secretory phase (Meduri et al., 2000). Together, these findings indicate that significant angiogenesis occurs during the proliferative phase of the menstrual cycle.

\section{Intravascular VEGF for non-sprouting angiogenesis in endometrium}

It is possible that the source of angiogenic factors for non-sprouting angiogenesis (intussusception or elongation), in which endothelial cells proliferate and migrate within vessels, is derived from cells within the vasculature rather than the tissues. In support of such a hypothesis is the demonstration that some circulating leucocytes, such as neutrophils, constitutively express VEGF in specific granules which are released when these cells become activated (Gaudry et al., 1997; Taichman et al., 1997). Other leucocytes, such as monocytes and $\mathrm{T}$ lymphocytes, produce VEGF when activated (Freeman et al., 1995; Harmey 

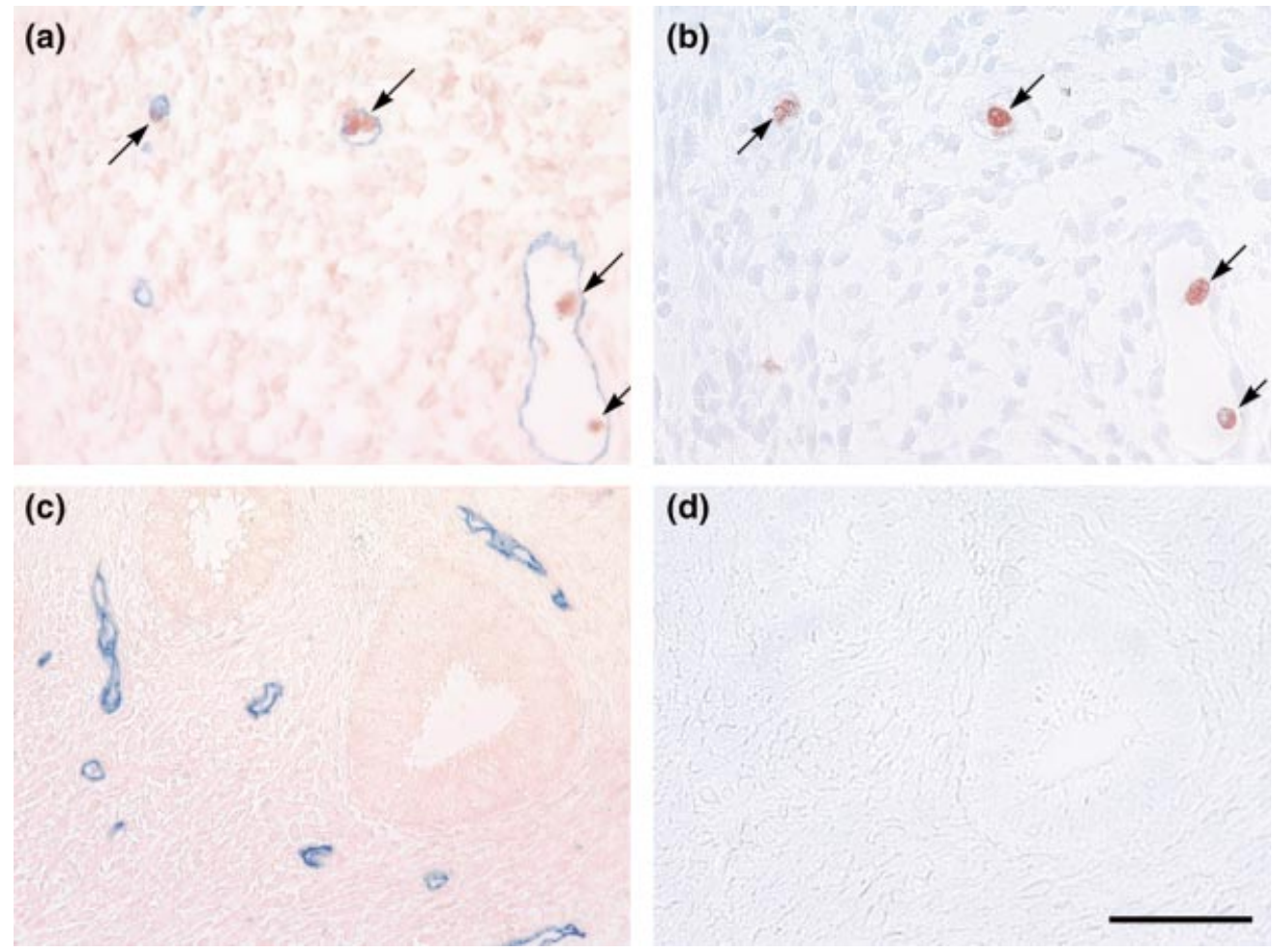

Fig. 2. Focal vascular endothelial growth factor (VEGF) expression in the human endometrium. Midproliferative stage functionalis $(a, b)$ and basalis $(c, d)$ from the same tissue double-immunostained with VEGF (red) and CD34 (blue) (a,c). Same field of view of serial sections $(3 \mu \mathrm{m})$ immunostained with anti-human neutrophil elastase (b,d) showing that focal VEGF in circulating leucocytes closely associated with endothelial cells of microvessels (arrows) is localized in adherent and marginating neutrophils (arrows). Significantly more VEGF-immunoreactive vessel-associated neutrophils were observed in the functionalis (and subepithelial capillary plexus) compared with the basalis. Scale bars represent $50 \mu \mathrm{m}$.

et al., 1998). There is evidence that in angiogenesis associated with inflammatory conditions, atherosclerosis and malignancy, extravasated leucocytes are a source of VEGF (Taichman et al., 1997; Harmey et al., 1998; Ramos et al., 1998). It is therefore possible that focal VEGF observed in human endometrium is located in intravascular leucocytes, which may have a role in endometrial angiogenesis by delivering VEGF directly to endothelial cells in vessels undergoing angiogenesis by non-sprouting mechanisms. We recently examined this hypothesis using full thickness endometrial sections, double immunohistochemical techniques and a morphometric approach (C. E. Gargett, F. Lederman, B. Heryanto, L. Gambino and P. A. W. Rogers, unpublished). In this study, the percentage of proliferating vessels in the subepithelial capillary plexus, functionalis and basalis was determined using anti-CD34 to mark endothelial cells and anti-proliferating cell nuclear antigen (anti-PCNA) to mark proliferating nuclei (Gargett et al., 1999). The percentage of vessel profiles containing VEGFimmunostained cells was determined in the same fields of view of serial sections double immunostained for CD34 and VEGF (Fig. 2a,c). The foci of VEGF associated with microvessels were identified as leucocytes rather than endothelial cells and, more specifically, as marginating and adherent neutrophils, as revealed by comparison with $3 \mu \mathrm{m}$ serial sections immunostained with neutrophil elastase antibodies (Fig. 2). Significantly more VEGF-immunoreactive vessel-associated neutrophils were observed in proliferative compared with secretory endometrium, for each of the subepithelial capillary plexus, functionalis and basalis regions. There was a gradient of VEGF-expressing microvessel-associated neutrophils through the depth of the endometrium; most of these cells were in the subepithelial plexus, followed by the functionalis and fewest in the basalis (compare Fig. 2a,b with c,d). In the proliferative phase, up to $75 \%$ of proliferating vessels in the subepithelial plexus and $46 \%$ of those in the functionalis contained marginating or adherent VEGF-expressing neutrophils. The most significant finding of this study was that a highly significant relationship between VEGF and endothelial cell proliferation in human endometrium was demonstrated for the first time (C. E. Gargett, F. Lederman, B. Heryanto, L. Gambino and P. A. W. Rogers, unpublished). Thus, the VEGF that correlated with endometrial angiogenesis was 
found in marginating and adherent neutrophils predominantly during the proliferative stages of the menstrual cycle in the subepithelial plexus and functionalis. Some focal microvessel VEGF was also present during the early secretory stage, but diminished with progression of the cycle. There have been other reports of neutrophils within microvessels in human endometrium, predominantly in the proliferative stages (Song et al., 1996). In contrast, few VEGF-expressing neutrophils were observed in the stroma in the proliferative and secretory stages, whereas many were in the stroma of menstrual endometrium as reported by Poropatich et al. (1987), but these neutrophils were not associated with microvessels and did not correlate with endothelial cell proliferation. From this study, we suggest the novel hypothesis that marginating and adherent neutrophils provide an intravascular source of VEGF for endometrial vessels undergoing angiogenesis by elongation or intussusception (Fig. 3).

\section{Neutrophil VEGF in other models of angiogenesis}

Intravascular neutrophils have been implicated in several models of angiogenesis, and have been particularly associated with acute and chronic inflammation. In an elegant mouse model of chronic airway inflammation, endothelial cell proliferation and vessel growth and enlargement were associated with leucocytes adherent to, and transmigrating through, microvessel walls (Murphy et al., 1999). Vessel growth was not by classical angiogenesis but rather through microvascular remodelling in which endothelial cells proliferated within intact vessel walls (Murphy et al., 1999). However, the role of leucocyte VEGF in vessel growth was beyond the scope of the study. In another study, neutrophils within or emigrating from microvessels stained strongly for VEGF in a variety of human mucosal chronic inflammatory lesions (Taichman et al., 1997). Although endothelial cell proliferation was not examined in these vessels, it was speculated that neutrophilderived VEGF was an efficient way of delivering VEGF to endothelial cells and it was concluded that neutrophils have a role in angiogenesis associated with inflammation (Taichman et al., 1997). Intravascular neutrophils and monocytes also play an important role in the neovascularization associated with diabetic retinopathy, in which large numbers of these cells have been documented in retinal microvessels (Schroder et al., 1991). The similarity of the above studies and our own observations in human endometrium raises the question as to whether endometrial angiogenesis has parallels to an inflammatory angiogenic response.

\section{Conclusions}

In summary, our studies show that focal VEGF correlates temporally and spatially with physiological angiogenesis in the human endometrium and appears to have a role in the development of the subepithelial capillary plexus and

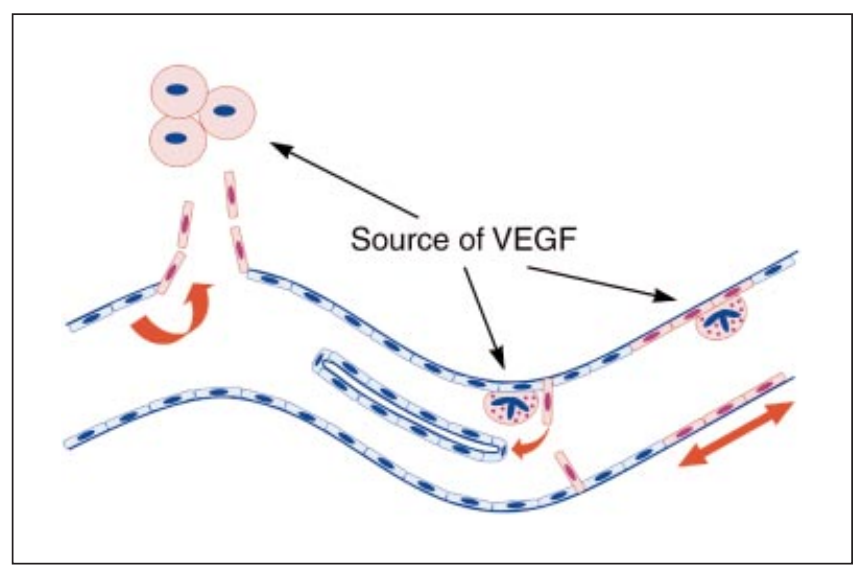

Fig. 3. Proposed hypothesis for endometrial angiogenesis. Marginating and adherent neutrophils provide an intravascular source of vascular endothelial growth factor (VEGF) for endometrial vessels undergoing angiogenesis by intussusceptive or elongation mechanisms, in contrast to tissue cells (for example stromal, luteal or tumour), which are a source of VEGF for vessels undergoing sprouting angiogenesis.

functionalis microvessels during the proliferative phase of the menstrual cycle. These studies also show that focal VEGF is located in neutrophils associated with microvessel walls. Furthermore, we propose the novel hypothesis that neutrophils are an intravascular source of the angiogenic growth factor VEGF for vessels that may grow by intussusception and elongation. This hypothesis provides a conceptual framework for the design of future studies to elucidate the role of focal microvessel VEGF in physiological angiogenesis of the human endometrium.

\section{References}

Key references are identified by asterisks.

Asahara T, Takahashi T, Masuda H, Kalka C, Chen DH, Iwaguro H, Inai Y, Silver M and Isner JM (1999) VEGF contributes to postnatal neovascularization by mobilizing bone marrow-derived endothelial progenitor cells EMBO Journal 18 3964-3972

Bausero P, Cavaillé F, Méduri G, Freitas S and Perrot-Applant M (1998) Paracrine action of vascular endothelial growth factor in the human endometrium: production and target sites, and hormonal regulation Angiogenesis 2 167-182

Ferrara N and Davis-Smyth T (1997) The biology of vascular endothelial growth factor Endocrine Reviews 18 4-25

Freeman MR, Schneck FX, Gagnon ML, Corless C, Soker S, Niknejad K, Peoples GE and Klagsbrun M (1995) Peripheral blood T lymphocytes and lymphocytes infiltrating human cancers express vascular endothelial growth factor: a potential role for T cells in angiogenesis Cancer Research 55 4140-4145

Gannon BJ, Carati CJ and Verco CJ (1997) Endometrial perfusion across the normal human menstrual cycle assessed by laser Doppler fluxmetry Human Reproduction 12 132-139

*Gargett CE, Lederman F, Lau TM, Taylor NH and Rogers PAW (1999) Lack of correlation between vascular endothelial growth factor production and endothelial cell proliferation in the human endometrium Human Reproduction 14 2080-2088

*Gaudry M, Bregerie O, Andrieu V, El Benna J, Pocidalo MA and Hakim J (1997) Intracellular pool of vascular endothelial growth factor in human neutrophils Blood 90 4153-4161 
Hanahan D (1997) Signaling vascular morphogenesis and maintenance Science 277 48-50

Hanahan D and Folkman J (1996) Patterns and emerging mechanisms of the angiogenic switch during tumorigenesis Cell 86 353-364

Harmey JH, Dimitriadis E, Kay E, Redmond HP and Bouchierhayes D (1998) Regulation of macrophage production of vascular endothelial growth factor (VEGF) by hypoxia and transforming growth factor $\beta-1$ Annals of Surgical Oncology 5 271-278

*Hii LLP and Rogers PAW (1998) Endometrial vascular and glandular expression of integrin $\alpha_{v} \beta_{3}$ in women with and without endometriosis Human Reproduction 13 1030-1035

Hornung D, Lebovic DI, Shifren JL, Vigne JL and Taylor RN (1998) Vectorial secretion of vascular endothelial growth factor by polarized human endometrial epithelial cells Fertility and Sterility 69 909-915

Iruela-Arispe ML, Rodriguez-Manzaneque JC and Abu-Jawdeh G (1999) Endometrial endothelial cells express estrogen and progesterone receptors and exhibit a tissue specific response to angiogenic growth factors Microcirculation 6 127-140

Meduri G, Bausero P and Perrot-Applanat M (2000) Expression of vascular endothelial growth factor receptors in the human endometrium: modulation during the menstrual cycle Biology of Reproduction 62 439-447

*Murphy TJ, Thurston G, Ezaki T and McDonald DM (1999) Endothelial cell heterogeneity in venules of mouse airways induced by polarized inflammatory stimulus American Journal of Pathology 155 93-103

Porapatich C, Rojas M and Silverberg SG (1987) Polymorphnuclear leuko- cytes in the endometrium during the normal menstrual cycle International .Journal of Gynecological Pathology 6 230-234

Ramos MA, Kuzuya M, Esaki T, Miura S, Satake S, Asai T, Kanda S, Hayashi T and Iguchi A (1998) Induction of macrophage VEGF in response to oxidized LDL and VEGF accumulation in human atherosclerotic lesions Arteriosclerosis, Thrombosis and Vascular Biology 18 1188-1196

Risau W (1997) Mechanisms of angiogenesis Nature 368 671-674

*Rogers PAW and Gargett CE (1999) Endometrial angiogenesis Angiogenesis 2 287-294

Schroder S, Palinski W and Schmid-Schönbein GW (1991) Activated monocytes and granulocytes, capillary nonperfusion, and neovascularization in diabetic retinopathy American Journal of Pathology 139 81-100

Smith SK (1998) Angiogenesis, vascular endothelial growth factor and the endometrium Human Reproduction Update 4 509-519

Song JY, Russell P, Markham R, Manconi F and Fraser IS (1996) Effects of high dose progestogens on white cells and necrosis in human endometrium Human Reproduction 11 1713-1718

*Taichman NS, Young S, Cruchley AT, Taylor P and Paleolog E (1997) Human neutrophils secrete vascular endothelial growth factor Journal of Leukocyte Biology 62 397-400

Torry DS and Torry RJ (1997) Angiogenesis and the expression of vascular endothelial growth factor in endometrium and placenta American Journal of Reproductive Immunology 37 21-29 